\title{
Allocation of Active Power Reserve from Active Distribution Networks Using a Cost-Benefit Approach: Application to Swissgrid Network
}

\author{
Mohsen Kalantar-Neyestanaki ${ }^{1}$, Mokhtar Bozorg ${ }^{1}$, Fabrizio Sossan ${ }^{1,2}$, Rachid Cherkaoui ${ }^{1}$ \\ Power Systems Group (PWRS) ${ }^{1}$ \\ Ecole Polytechnique Federale de Lausanne (EPFL) \\ Lausanne, Switzerland. \\ PERSEE $^{2}$ \\ Mines ParisTech \\ France. \\ \{mohsen.kalantar, mokhtar.bozorg, fabrizio.sossan, rachid.cherkaoui\}@epfl.ch fabrizio.sossan@mines-paristech.fr
}

\begin{abstract}
The flexibility of distributed energy resources (DERs) accommodated in active distribution networks (ADNs) can be aggregated and then used to provide ancillary services to the transmission system. In this context, this paper presents a linear optimization method for the transmission system operator (TSO) to allocate its required active power reserve from aggregated resources installed in active distribution systems (ARADSs) as well as dispatchable bulk power plants (DBPPs). It consists in a linear optimization problem that minimizes the sum of the expected cost of active power reserve allocated from all possible providers (including ARADSs and DBPPs) and the expected cost of load not served over a desired time horizon. The value of lost load (VOLL) index is used as a criterion to realize an economical balance between the expected cost of allocated reserve and expected cost of load not served. The method leverages scenarios of power system contingencies and forecast errors of loads and renewable generation to represent typical operational uncertainties. A simulation proofof-concept using real-data from the transmission system operator of Switzerland, Swissgrid, is provided to illustrate the performance of the method.
\end{abstract}

Index Terms--Active distribution network (ADN), active power reserve, aggregated resources of active distribution system (ARADS), energy storage system (ESS), distributed energy resource (DER).

\section{NOMENCLATURE}

Abbreviations

ADN Active Distribution Network.

ARADS Aggregated Resources of Active Distribution

DER System.

Distributed Energy Resource.

DG Distributed Generator.

DBPP Dispatchable Bulk Power Plant.

ESS Energy Storage System.

FL Flexible Load.

SPP Stochastic Power Plant.

TSO Transmission System Operator.

VOLL Value of lost load.

Indices and Sets

$l \quad$ Index for loads.

$h$ Index for ARADSs.

$i, j \quad$ Index for buses of the transmission system.

$k$ Index for power plants including both DBPPs and

$k$ SPPs.

$s \quad$ Index for scenario.

$t, \tau$ Index for time intervals in hour.

$\mathbb{B}$ Set of buses of the transmission system.
$\mathbb{B}_{i} \quad$ Set of adjacent buses connected through transmission

$\mathbb{B}_{i} \quad$ lines to bus $i$.

A Set of ARADSs connected to the transmission system.

$\mathbb{A}_{i} \quad$ Set of ARADSs connected to bus $i$.

$\mathbb{D} \mathbb{G}$ Set of DBPPs connected to the transmission system.

$\mathbb{D G}_{i}$ Set of DBPPs connected to bus $i$.

$\mathbb{L}_{i} \quad$ Set of loads connected to bus $i$.

$\mathbb{S G}_{i}$ Set of SPPs connected to bus $i$.

$\mathbb{S}$ Set of scenarios.

$\mathbb{T}$ Set of time intervals of the scheduling time horizon.

Variables

$E_{h}^{\mathrm{PE}+}$ Upward/downward booked energy capacity from

$E_{h}^{\mathrm{PE}+}, \quad \mathrm{P} \& \mathrm{E}-\mathrm{constrained}$ active power reserve of ARADS $h$ to

$E_{h}^{\mathrm{PE}-} \quad$ the transmission system [p.u.].

$P_{\text {hts }}^{\text {SL }}, \quad$ Shed load of ARADS $h /$ load $l$ during time interval $t$ $P_{l t s}^{\mathrm{SL}} \quad$ for scenario $s$ [p.u.].

$r_{\text {G+ }}$ Upward/downward active power reserve deployed

$r_{k t s}^{G}$, from DBPP $k$ during time interval $t$ for scenario $s$

$r_{k t s}^{\mathrm{G}-} \quad$ [p.u.].

$r^{\mathrm{P}+} \quad$ Upward/downward P-constrained active power $r_{\text {hts }}^{\mathrm{P}}$, reserve deployed from ARADS $h$ to the transmission $r_{\text {hts }}^{\mathrm{P}-} \quad$ system during time interval $t$ for scenario $s$ [p.u.].

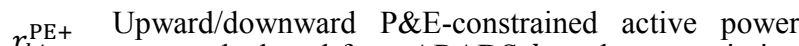
$r_{\text {hts }}^{\mathrm{PE}}$, reserve deployed from ARADS $h$ to the transmission $r_{\text {hts }}^{\mathrm{PE}-} \quad$ system during time interval $t$ for scenario $s$ [p.u.].

$R_{k}^{\mathrm{G}+}$, Upward/downward booked active power reserve $R_{k}^{\mathrm{G}-} \quad$ capacity from DBPP $k$ [p.u.].

$R_{h}^{\mathrm{P}+}$, Upward/downward booked power capacity from P-

$R_{h}^{\mathrm{P}}{ }^{\mathrm{P}}$,
$R_{h}^{\mathrm{P}}$$\quad$ constrained active power reserve of ARADS $h$ to the $R_{h}^{\mathrm{P}-} \quad$ transmission system [p.u.].

$R_{h}^{\mathrm{PE}+}$ Upward/downward booked power capacity from P\&E-

$R_{h}^{\mathrm{PE}-}$ ' constrained active power reserve of ARADS $h$ to the $R_{h}^{\mathrm{PE}-} \quad$ transmission system [p.u.].

$\delta_{i t s} \quad$ Voltage angle of bus $i$ during time interval $t$ for scenario $s$ [rad].

Parameters

$A_{\text {G }}^{G} \quad$ Binary 0,1 parameter denoting the on-off state of

$\mathrm{A}_{k t s}^{\mathrm{G}} \quad$ DBPP $k$ during time interval $t$ for scenario $s$.

Binary 0,1 parameter denoting the availability-

$\mathrm{A}_{i j t s}^{\mathrm{T}} \quad$ unavailability of the transmission line between bus $i$ and bus $j$ during time interval $t$ for scenario $s$.

$B_{i j} \quad$ Longitudinal susceptance of the transmission line $\mathrm{B}_{i j} \quad$ between bus $i$ and bus $j$ [p.u.].

$\mathrm{C}_{h}^{\mathrm{E}+}, \quad$ Price of the upward/downward energy capacity of $\mathrm{C}_{h}^{\mathrm{E}-} \quad$ P\&E-constrained active power reserve of ARADS 


\section{$h[\mathrm{CHF} / \mathrm{p} . \mathrm{u}$.$] .$}

$\mathrm{C}_{k}^{\mathrm{G}+}$, Price of the upward/downward active power $\mathrm{C}_{k}^{\mathrm{G}-}{ }^{\prime} \quad$ reserve capacity for DBPP $k$ [CHF/p.u.].

$\mathrm{C}_{h}+\quad$ Price of the upward/downward power capacity of $\mathrm{P}-$ Constrained active power reserve of ARADS $h$ $\mathrm{C}_{h}^{\mathrm{P}-} \quad[\mathrm{CHF} /$ p.u. $]$.

$\mathrm{C}_{h}^{\mathrm{PE}+} \quad$ Price of the upward/downward power capacity of PE-' P\&E-constrained active power reserve of ARADS $\mathrm{C}_{h}^{\mathrm{PE}-} \quad h[\mathrm{CHF} /$ p.u. $]$.

$F^{\max } \quad$ Maximum power flow limit of the transmission line $\mathrm{F}_{i j} \quad$ between buses $i$ and $j$ [p.u.].

$\mathrm{P}_{h t}, \quad$ Active power schedule of ARADS $h$ /power plant $k$ $\mathrm{P}_{k t}, \mathrm{P}_{l t}$ load $l$ during time interval $t$ [p.u.].

$\Delta \mathrm{P}_{h t s}, \quad$ Deviation from scheduled active power for ARADS $\Delta \mathrm{P}_{k t s}, \quad h / \mathrm{SPP} k /$ load $l$ during time interval $t$ and scenario $\Delta \mathrm{P}_{\text {lts }} \quad s$ [p.u.].

$\mathrm{P}_{k}^{\mathrm{Gmax}}$, Maximum and minimum output power of power $\mathrm{P}_{k}^{\text {Gmin }}$ ' plant $k$ [p.u.].

Rated power of the substation connecting ARADS $\mathrm{T}_{h}, \mathrm{~T}_{k} \quad h$ /power plant $k$ to the transmission system [p.u.].

VOLL $_{i} \quad[\mathrm{CHF} /$ p.u. $]$.

$\pi_{k}^{\mathrm{G}+}$, Price of the upward/downward active power $\pi_{k}^{\mathrm{G}-} \quad$ reserve provided by DBPP $k$ [CHF/p.u.].

$\pi_{h}^{\mathrm{P}+}, \quad$ Price of the upward/downward P-constrained active $\pi_{h}^{\mathrm{P}-} \quad$ power reserve provided by ARADS $h$ [CHF/p.u.].

$\pi_{h}^{\mathrm{PE}+} \quad$ Price of the upward/downward P\&E-constrained ' $\pi_{h}^{\mathrm{PE}-} \quad$ [CHF/p.u.].

$\rho_{s} \quad$ Probability of occurrence of scenario $s$.

\section{INTRODUCTION}

Environmental concerns and recent developments in renewable energy technologies are leading towards replacing conventional generation in favor of production from renewables. For instance, in Switzerland, governmental goals have set to phase out nuclear generation by 2050, thus opening the way to production from distributed energy resources (DERs) [1]. The main challenge of this transition is the increasing demand for controllable resources that need to be deployed to compensate for the intermittent characteristic of renewable generation and guarantee the frequency/voltage regulation, power quality, and congestion management [2]-[4].

The number of DERs like dispatchable distributed generators (DGs, e.g., gas turbines, micro turbines, combined heat and power units) and energy storage systems (ESSs, e.g., batteries, fuel-cells), in existing distribution networks is expected to increase steeply in the near future [4]. Moreover, the increasing deployment of monitoring and communication infrastructures in distribution systems [5] is envisaged to facilitate the adoption of new automated control strategies for active distribution networks (ADNs), thereby allowing DERs together with the demand side management of flexible loads (e.g., thermostatically controlled loads) to be aggregated for providing ancillary services to both distribution and transmission grids. In this emerging architecture, the main question that arises is how much are the active power reserves that a transmission system operator
(TSO) should book from all reserve providers to minimize its total cost consisting on the expected cost of the load not served and allocated reserve over a desired time horizon (e.g., 1 day or 1 week)?

Traditional practices for allocating reserve consist in scheduling the active power reserve required by the TSO while considering only dispatchable bulk power plants (DBPPs, e.g., gas, hydro, thermal power plants) and not considering generally the potential of ADNs, see for instance [6]-[14]. Traditional methods can be categorized into deterministic and probabilistic. A deterministic approach sets the required amount of active power reserve larger than or equal to the capacity of the largest online unit [7]. As far as the probabilistic approach is concerned, two mainstream methodologies can be traced in the existing literature: statistic-based and optimization-based methods. The former relies on a statistical assessment to characterize the contingencies and uncertainties that lead to unbalances (e.g., power plants/transmission lines outages, forecast errors of demand and renewable generation), whereby they determine the reserve so that a target risk level is satisfied (e.g., [8][10]). The latter incorporates the risk index into an optimization problem, and then the optimum amount of required active power reserve is achieved by solving that problem (e.g., [11]-[14]). Methods proposed in [13] and [14] minimize the total cost of TSO by making a balance between the cost of reserve allocation and cost of load not served, whereas methods in [11] and [12] aim at either minimizing only the cost of reserve allocation or satisfying only a risk index like LOLP (loss of load probability) and ELNS (expected load not served).

All the above-mentioned methods may lead to excessive or deficient amount of reserve so they are suboptimal since:

- they do not account for the capability of ADNs for provision of active power reserve;

- all of them, except for [13] and [14], don't make a balance between the cost of reserve allocation and cost of load not served.

in other words, the amount of booked reserve through these methods are not economically/technically justifiable. The above-mentioned limitations found in [6]-[14] paved the way to the method developed in this paper. In [15], the authors of the present paper introduced the concept of the aggregated resources of active distribution systems (ARADSs) with the aim of reserve provision to the transmission system. They first defined an ARADS as the aggregated flexibilities model of DERs located in an ADN, then, established a framework for modeling ARADSs as seen from TSO perspective. This present paper considers the capability of ARADSs for provision of active power reserve to the TSO. It leverages the ARADS modeling framework proposed in [15] and a cost-benefit approach to develop a method for allocating the required active power reserved of TSO from both DBPPs and ARADSs while considering line/generation outages as well as forecast errors of the loads and stochastic generation (scenarios). This method minimizes the total cost of TSO throughout the scheduling horizon using a multi-period (multi-scenario) optimization method. Thus, it can trade the expected load not served of the network among time intervals, i.e. it may book less reserve and accept a higher load not serve at some time intervals in such a way that the total cost of TSO over the total scheduling 


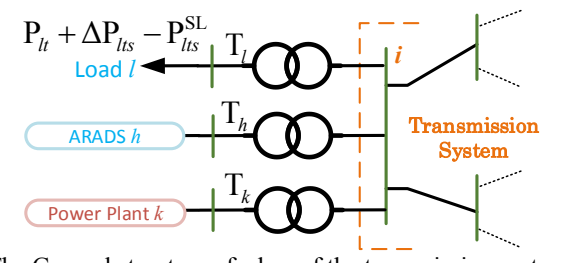

Fig. 1. The General structure of a bus of the transmission system.

time horizon is minimized. Although the proposed formulation is generic and can accommodate any scheduling horizon, it is considered here to perform day-ahead scheduling to comply with conventional scheduling/ operational paradigm of current power systems. To the best knowledge of the authors, this problem and its treatment have not been reported in the literature.

The rest of the paper is organized as follows: Section II models the power system as seen from TSO perspective. Section III presents the problem formulation. To illustrate the effectiveness of the proposed approach, Section IV gives the numerical results considering the Swissgrid network case. Finally, conclusions are presented in Section V.

\section{POWER System Modeling}

This section models the power system as seen from a TSO's perspective with reliance on the following modelling assumptions:

- only active power and active power reserve are taken into consideration;

- the transmission system is loss-less and modeled by DC power flow equations;

- the scheduling time horizon and dispatching time intervals are 24 hours and 1 hour, respectively. However, it can vary without losing the generality of the problem;

- the hourly active power schedule of the power plants/ARADSs and loads are known $\left(\mathrm{P}_{k t}, \mathrm{P}_{h t}, \mathrm{P}_{l t}\right)$;

- the uncertainties of loads, ARADSs and stochastic power plants (SPPs) are modeled as stochastic power sources or loads connected to the transmission system $\left(\Delta \mathrm{P}_{l t s}, \Delta \mathrm{P}_{h t s}, \Delta \mathrm{P}_{k t s}\right)$.

The general structure of a bus of the transmission system is illustrated in Fig. 1. It shows $\operatorname{ARADS} h$, power plant $k$, and load $l$ connected to the transmission system through 3 substations. In the rest of this section, the models of ARADSs and power plants are introduced.

\section{A. ARADS Modeling}

The flexibility of DERs can be exploited for provision of active power reserve to the transmission system. However, there are several obstacles in the way to achieve this goal as:

- DERs usually have small capacities;

- DERs typically belong to different owners;

- neither TSO nor distribution system operator generally has access to the DERs' data and resources.

To resolve these obstacles, the authors in [15] introduced a novel entity called ARADS under the management of a third market player called aggregator. From TSO perspective, each ARADS can be modeled as an equivalent load along with a P-constrained (i.e. power constrained) and a P\&E-constrained (i.e. power and energy constrained) resources of active power reserve, as shown in Fig. 2. The

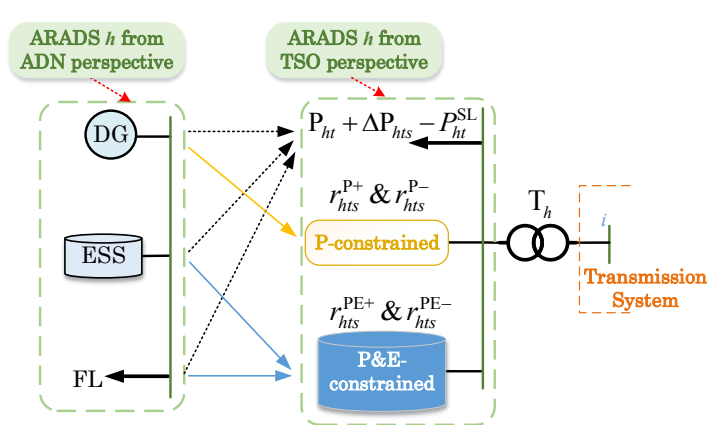

Fig. 2. The general structure of ARADS $h$ connected to bus $i$. The notation refers to time interval $t$ and scenario $s$ [15].

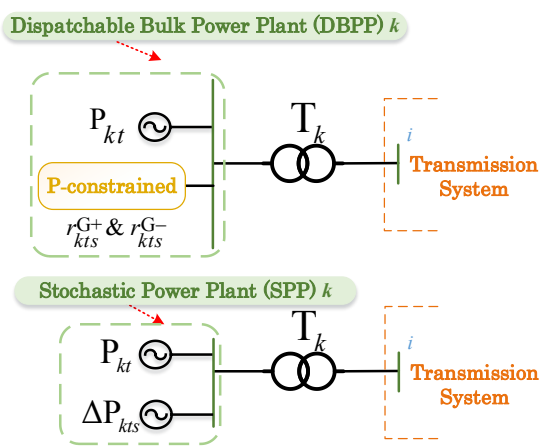

Fig. 3. The model of power plant $k$ connected to bus $i$, from TSO point of view. The notation refers to time interval $t$ and scenario $s$.

equivalent load represents the net active power schedule for the ARADS. P-constrained resources are restricted only by the active power capacity as in the case of dispatchable DGs. The P\&E-constrained resources are restricted by both active power and energy capacity as in the case of ESSs and FLs. For more details, please refer to [15].

\section{B. Power Plant Modeling}

The power plants are categorized into dispatchable and stochastic (i.e., non-dispatchable). During the real time operation, DBPPs follow their pre-defined (typically dayahead) scheduled plan and also provide reserve to the TSO, while SPPs, like solar and wind power plants, may not follow their forecasted schedules due to predictions errors and they are sources of uncertainties. In line with the classification of the active power reserve resources presented in II.A, the power plants can be modeled as illustrated in Fig. 3.

\section{SCENARIO-BASED STOCHASTIC OPTIMIZATION PROBLEM FORMULATION}

Based on the models for ARADSs and power plants described in the previous section and exploiting the DC power flow model [7] along with a cost-benefit approach, the optimal allocation of active power reserve for a TSO from DBPPs and ARADSs is formulated as the following linear programming problem:

$\min _{\Psi} C^{\text {Interruption }}(\Psi)+C^{\text {Reserve }}(\Psi)$,

subject to:

$0 \leq r_{k t s}^{\mathrm{G}+} \leq \mathrm{A}_{k t s}^{\mathrm{G}} R_{k}^{\mathrm{G}+} \quad \forall k \in \mathbb{D} \mathbb{G}, \forall t \in \mathbb{T}, \forall s \in \mathbb{S}$,

$0 \leq r_{k t s}^{\mathrm{G}-} \leq \mathrm{A}_{k t s}^{\mathrm{G}} R_{k}^{\mathrm{G}-} \quad \forall k \in \mathbb{D} \mathbb{G}, \forall t \in \mathbb{T}, \forall s \in \mathbb{S}$,

$0 \leq R_{k}^{\mathrm{G}+} \leq \mathrm{P}_{k}^{\mathrm{Gmax}}-\mathrm{P}_{k t} \quad \forall k \in \mathbb{D} \mathbb{G}, \forall t \in \mathbb{T}$,

$0 \leq R_{k}^{\mathrm{G}-} \leq \mathrm{P}_{k t}-\mathrm{P}_{k}^{\mathrm{Gmin}} \quad \forall k \in \mathbb{D} \mathbb{G}, \forall t \in \mathbb{T}$, 


$$
\begin{aligned}
& 0 \leq r_{h t s}^{\mathrm{P}+} \leq R_{h}^{\mathrm{P}+} \\
& 0 \leq r_{h t s}^{\mathrm{P}-} \leq R_{h}^{\mathrm{P}-} \\
& 0 \leq r_{h t s}^{\mathrm{PE}+} \leq R_{h}^{\mathrm{PE}+} \\
& 0 \leq r_{h t s}^{\mathrm{PE}-} \leq R_{h}^{\mathrm{PE}-} \\
& -E_{h}^{\mathrm{PE}-} \leq \sum_{\tau=1}^{t}\left(r_{h \tau s}^{\mathrm{PE}+}-r_{h \tau s}^{\mathrm{PE}-}\right) \leq E_{h}^{\mathrm{PE}+} \\
& \forall h \in \mathbb{A}, \forall t \in \mathbb{T}, \forall s \in \mathbb{S}, \\
& \forall h \in \mathbb{A}, \forall t \in \mathbb{T}, \forall s \in \mathbb{S} \text {, } \\
& \forall h \in \mathbb{A}, \forall t \in \mathbb{T}, \forall s \in \mathbb{S} \text {, } \\
& \forall h \in \mathbb{A}, \forall t \in \mathbb{T}, \forall s \in \mathbb{S} \text {, } \\
& \forall h \in \mathbb{A}, \forall t \in \mathbb{T}, \forall s \in \mathbb{S}, \\
& 0 \leq R_{h}^{\mathrm{P}+} \leq \mathrm{R}_{h}^{\mathrm{P}+\text { max }} \\
& \forall h \in \mathbb{A} \text {, } \\
& 0 \leq R_{h}^{\mathrm{P}-} \leq \mathrm{R}_{h}^{\mathrm{P}-\text {, max }} \\
& \forall h \in \mathbb{A} \text {, } \\
& 0 \leq R_{h}^{\mathrm{PE}+} \leq \mathrm{R}_{h}^{\mathrm{PE}+\text {,max }} \\
& \forall h \in \mathbb{A} \text {, } \\
& 0 \leq R_{h}^{\mathrm{PE}-} \leq \mathrm{R}_{h}^{\mathrm{PE}-\text {, } m a x} \\
& \forall h \in \mathbb{A} \text {, } \\
& 0 \leq E_{h}^{\mathrm{PE}+} \leq \mathrm{E}_{h}^{\mathrm{PE}+\text {,max }} \\
& \forall h \in \mathbb{A} \text {, } \\
& 0 \leq E_{h}^{\mathrm{PE}-} \leq \mathrm{E}_{h}^{\mathrm{PE}-, \text { max }} \quad \forall h \in \mathbb{A} \text {, } \\
& -\mathrm{T}_{h} \leq-\left(\mathrm{P}_{h t}+\Delta \mathrm{P}_{h t s}-P_{h t s}^{\mathrm{SL}}\right)+r_{h t s}^{\mathrm{P}+}-r_{h t s}^{\mathrm{P}-}+r_{h t s}^{\mathrm{PE}+} \\
& -r_{h t s}^{\mathrm{PE}-} \leq \mathrm{T}_{h} \quad \forall h \in \mathbb{A}, \forall t \in \mathbb{T}, \forall s \in \mathbb{S}, \\
& -\mathrm{T}_{k} \leq \mathrm{A}_{k t s}^{\mathrm{G}}\left(\mathrm{P}_{k t}+r_{k t s}^{\mathrm{G}+}-r_{k t s}^{\mathrm{G}-}\right) \leq \mathrm{T}_{k} \\
& \forall k \in \mathbb{D} \mathbb{G}, \forall t \in \mathbb{T}, \forall s \in \mathbb{S}, \\
& -\mathrm{F}_{i j}^{\max } \leq \mathrm{A}_{i j t s}^{\mathrm{T}} \mathrm{B}_{i j}\left(\delta_{i t s}-\delta_{j t s}\right) \leq \mathrm{F}_{i j}^{\max }, \\
& \forall i, j \in \mathbb{B}, \forall t \in \mathbb{T}, \forall s \in \mathbb{S}, \\
& \sum_{h \in \mathbb{A}_{i}}\left[-\left(\mathrm{P}_{h t}+\Delta \mathrm{P}_{h t s}-P_{h t s}^{\mathrm{SL}}\right)+r_{h t s}^{\mathrm{P}+}-r_{h t s}^{\mathrm{P}-}+r_{h t s}^{\mathrm{PE}+}-r_{h t s}^{\mathrm{PE}-}\right]+ \\
& \sum_{k \in \mathbb{D} \mathbb{G}_{i}}\left[\mathrm{~A}_{k t s}^{\mathrm{G}}\left(\mathrm{P}_{k t}+r_{k t s}^{\mathrm{G}+}-r_{k t s}^{\mathrm{G}-}\right)\right]+\sum_{k \in \mathbb{S} \mathbb{G}_{i}}\left[\mathrm{~A}_{k t s}^{\mathrm{G}}\left(\mathrm{P}_{k t}+\Delta \mathrm{P}_{k t s}\right)\right] \\
& +\sum_{l \in \mathbb{L}_{i}}\left[-\left(\mathrm{P}_{l t}+\Delta \mathrm{P}_{l t s}-P_{l t s}^{\mathrm{SL}}\right)\right]=\sum_{j \in \mathbb{B}_{i}} \mathrm{~A}_{i j t s}^{\mathrm{T}} \mathrm{B}_{i j}\left(\delta_{i t s}-\delta_{j t s}\right) \\
& \forall i \in \mathbb{B}, \forall t \in \mathbb{T}, \forall s \in \mathbb{S},
\end{aligned}
$$

this set of variables comprises the deployed reserve and booked reserve (reserve capacity) from all DBPPs and ARADSs, as well as the state of the transmission system.

The objective function (1) aims at minimizing:

- $C^{\text {Interruption }}(\Psi)$ : the expected cost of load not served with respect to the realization of the set of credible scenarios which can be expressed as:

$C^{\text {Interruption }}(\Psi)=\sum_{i \in \mathbb{B}} \sum_{t \in \mathbb{T}} \sum_{s \in \mathbb{S}} \rho_{s} \operatorname{VOLL}_{i}\left[\sum_{l \in \mathbb{L}_{i}} P_{l t s}^{\mathrm{SL}}+\sum_{h \in \mathbb{A}_{i}} P_{h t s}^{\mathrm{SL}}\right]$.

It measures the benefit of reserve in terms of the reduction in the expected cost of load not served, i.e. it characterizes the benefit.

- $C^{\text {Reserve }}(\Psi)$ : the expected cost of active power reserve allocation (comprising cost of booked reserve capacity as well as cost of deployed reserve) from all providers including ARADSs and DBPPs over the scheduling time horizon (24 hours) over the set of credible scenarios. It characterize the cost of reserve. It can be expressed as:

$$
\begin{aligned}
& C^{\text {Reserve }}(\Psi)=\sum_{k \in \mathbb{D} G} \sum_{t \in \mathbb{T}} \sum_{s \in \mathbb{S}} \rho_{s}\left(\pi_{k}^{\mathrm{G}+} r_{k t s}^{\mathrm{G}+}+\pi_{k}^{\mathrm{G}-} r_{k t s}^{\mathrm{G}-}\right)+ \\
& \sum_{h \in \mathbb{A}} \sum_{t \in \mathbb{T}} \sum_{s \in \mathbb{S}} \rho_{s}\left(\pi_{h}^{\mathrm{P}+} r_{\text {hts }}^{\mathrm{P}+}+\pi_{h}^{\mathrm{P}-} r_{\text {hts }}^{\mathrm{P}-}+\pi_{h}^{\mathrm{PE}+} r_{h t s}^{\mathrm{PE}+}+\pi_{h}^{\mathrm{PE}-} r_{\text {hts }}^{\mathrm{PE}-}\right)
\end{aligned}
$$

$$
\begin{aligned}
& +\sum_{h \in \mathbb{A}}\left(\mathrm{C}_{h}^{\mathrm{P}+} R_{h}^{\mathrm{P}+}+\mathrm{C}_{h}^{\mathrm{P}-} R_{h}^{\mathrm{P}-}+\mathrm{C}_{h}^{\mathrm{PE}+} R_{h}^{\mathrm{PE}+}+\mathrm{C}_{h}^{\mathrm{PE}-} R_{h}^{\mathrm{PE}-}+\right. \\
& \left.\mathrm{C}_{h}^{\mathrm{E}+} E_{h}^{\mathrm{PE}+}+\mathrm{C}_{h}^{\mathrm{E}-} E_{h}^{\mathrm{PE}-}\right)+\sum_{k \in \mathbb{D} G}\left(\mathrm{C}_{k}^{\mathrm{G}+} R_{k}^{\mathrm{G}+}+\mathrm{C}_{k}^{\mathrm{G}-} R_{k}^{\mathrm{G}-}\right),
\end{aligned}
$$

where the first term is the expected (with respect to the realization of the set of scenarios) cost of the upward/downward deployed reserve from all DBPPs. The second term is the expected cost of the upward/downward deployed reserve from all ARADSs. This term includes two parts, the first part is related to the expected cost of the upward/downward deployed reserve from P-constrained resources of ARADSs, and the second part is related to the expected cost of the upward/downward deployed reserve from P\&E-constrained resources of ARADSs. The third term consists in two parts, the first part represents the cost of upward/downward booked reserve capacity from Pconstrained resources of ARADSs, while the second part represents the cost of upward/downward booked reserve capacity from P\&E-constrained resources of ARADSs. The fourth term represents the cost of upward/downward booked reserve capacity from all DBPPs.

The set of constraints (2)-(21) binds the objective function (1). Constraints (2) and (3) respectively model the power limits of the upward and downward P-constrained reserve provided by DBPPs. The set of constraints (4) and (5) enforces the upward and downward booked active power reserve capacities of DBPPs to respect the maximum and minimum active power limits of DBPPs. Constraints (6) and (7) respectively model the power limits of the upward and downward P-constrained reserve provided by ARADSs. Constraints (8) and (9) respectively model the power limits of the upward and downward P\&E-constrained reserve provided by ARADSs. The constraint (10) models the energy limits of the upward and downward P\&E-constrained reserve provided by the ARADSs. The set of constraints (11)-(16) enforces the upward/ downward booked Pconstrained and P\&E-constrained reserve capacities of ARADSs to respect the maximum reserve capacities offered by ARADSs to $\quad$ TSO, namely $\mathrm{R}_{h}^{\mathrm{P}+\text {, max }}, \mathrm{R}_{h}^{\mathrm{P}-, \text { max }}, \mathrm{R}_{h}^{\mathrm{PE}+\text {, } \max }, \mathrm{R}_{h}^{\mathrm{PE}-, \text { max }}, \quad \mathrm{E}_{h}^{\mathrm{PE}+\text {, max }}$ and $\mathrm{E}_{h}^{\mathrm{PE}-\text {, max }}$. The set of constraints (17) and (18) models the power limits of the substations connecting the ARADSs and DBPPs to the transmission system, respectively.

As the transmission system is assumed loss-less and modeled with the DC power flow model, the power flow in a transmission line is proportional to the difference of the voltage angle of the terminal buses of that line; so constraint (19) represents the power flow limits of the transmission lines. The constraint (20) enforces the power balance for all buses of the transmission system. The constraint (21) considers bus number 1 as the reference for the angle of the voltages.

The above-mentioned objective function along with the defined constraints build a scenario-based stochastic linear optimization formulation for the problem of optimal allocation of active power reserve from ARADSs and DBPPs. The outcome of this optimization problem determines the required optimal capacity of active power reserve from ARADSs and DBPPs such that the total cost of TSO is minimized. 


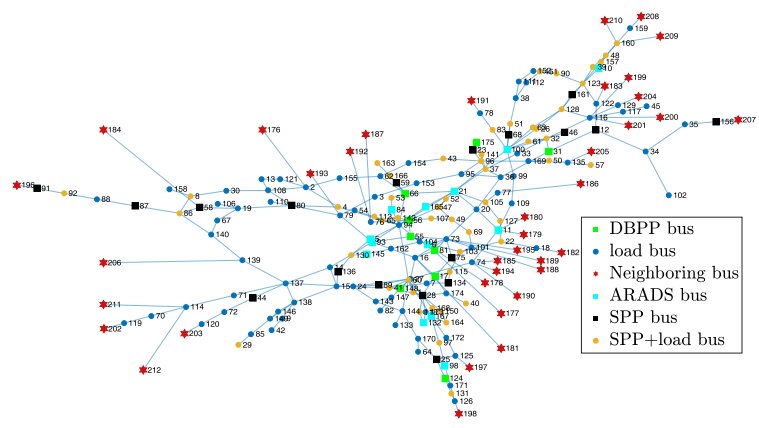

Fig. 4. Swissgrid network topology.

\section{RESULTS}

\section{A. Case Study}

The high-voltage transmission network of Switzerland is considered as the case study. The grid is operated by Swissgrid and is an important electricity pathway due to its location in central Europe. It includes 212 buses at $380 \mathrm{kV}$ and $220 \mathrm{kV}$ connected through 284 transmission lines and 25 high-voltage transformers. It is connected to France, Germany, Italy and Austria through 37 buses. In this work, the interconnections to the neighboring countries are modeled as a constant positive or negative injection at the connecting nodes during each time interval. Fig. 4 shows the network configuration. Its buses can be categorized into 6 types:

- DBPP buses, which interface DBPPs;

- Load buses, which interface aggregated consumers;

- Neighboring buses, which connect the network to neighboring TSOs;

- ARADS buses, which interface ARADSs;

- SPP buses, which interface SPPs;

- SPP + load buses: where both SPPs and loads are connected.

The topology, technical parameters and profile of the nodal generation/consumption of this case study are directly provided by Swissgrid. The prices of booked reserve capacity and deployed reserve of ARADSs and DBPPs are extracted from the Swissgrid reserve market clearing prices [16]. To address the correlation between the price and demand of reserve, all of these prices are modeled as staircase (piece-wise constant) functions of the amount of booked/deployed reserve.

\section{B. Contingencies and Uncertainties Modeling}

The share of stochastic generation is assumed $30 \%$ of total generation. Forced outage rates and mean time to repairs of all components including power plants and transmission lines/transformers are chosen based on statistics available in [17], [18]. During 24-hour time period of study, the state (availability/unavailability) of these components are modeled as independent two-states 0, 1 Markov chains. Then, sequential Monte Carlo simulation is used to provide scenarios that represent outages of generators, transmission lines and transformers. Moreover, the day-ahead forecast errors of loads and stochastic generation are modeled as independent Gaussian distributions. The forecast error of each load(/SPP) is sampled from a normal distribution with 0 mean and such that the standard deviation of the total $\operatorname{load}(/$ stochastic generation) forecast error is $3 \%(/ 8 \%)$ of the whole grid forecasted load(/stochastic generation) [19]. These forecast errors model deviation of the net power
Table I. Impact of VOLL on the cost and ELNS of the grid for case 1.

\begin{tabular}{c||c||c|c}
\hline \multicolumn{1}{c||}{ VOLL (CHF/MWh) } & 200 & 400 & 600 \\
\hline $\begin{array}{c}\text { Total expected cost of TSO } \\
(\mathrm{CHF} / \text { day) }\end{array}$ & $264^{\prime} 450$ & $388^{\prime} 800$ & $461^{\prime} 700$ \\
\hline ELNS (MWh/day) & 828 & 479 & 237 \\
\hline$\sum_{k \in \mathbb{D G}} R_{k}^{\mathrm{G}+}(\mathrm{MW} /$ day $)$ & 144 & 475 & 801 \\
\hline$\sum_{k \in \mathbb{D} G} R_{k}^{\mathrm{G}-}(\mathrm{MW} /$ day $)$ & 85 & 82 & 76 \\
\hline$\sum_{h \in \mathbb{A}} R_{h}^{\mathrm{P}+}(\mathrm{MW} /$ day $)$ & 232 & 365 & 365 \\
\hline$\sum_{h \in \mathbb{A}} R_{h}^{\mathrm{P}-}(\mathrm{MW} /$ day) & 145 & 140 & 136 \\
\hline$\sum_{h \in \mathbb{A}} R_{h}^{\mathrm{PE}+}(\mathrm{MW} /$ day $)$ & 9 & 11 & 21 \\
\hline$\sum_{h \in \mathbb{A}} R_{h}^{\mathrm{PE}-}(\mathrm{MW} /$ day $)$ & 152 & 159 & 169 \\
\hline$\sum_{h \in \mathbb{A}} E_{h}^{\mathrm{PE}+}(\mathrm{MWh} /$ day $)$ & 49 & 56 & 142 \\
\hline$\sum_{h \in \mathbb{A}} E_{h}^{\mathrm{PE}-}(\mathrm{MWh} /$ day $)$ & 103 & 138 & 143 \\
\hline $\begin{array}{c}\text { Total expected deployed reserve } \\
(\mathrm{MWh} / \text { day) }\end{array}$ & $2{ }^{\prime} 173$ & $2{ }^{\prime} 522$ & $2{ }^{\prime} 766$ \\
\hline
\end{tabular}

Table II. Impact of VOLL on the cost and ELNS of the grid for case 2. VOLL (CHF/MWh)

\begin{tabular}{c||c|c|c}
\hline \begin{tabular}{c||} 
Total expected cost of TSO \\
$(\mathrm{CHF} /$ day $)$
\end{tabular} & 200 & 400 & 600 \\
\hline ELNS (MWh/day) & 917 & 763 & 527 \\
\hline$\sum_{k \in \mathbb{D G}} R_{k}^{\mathrm{G}+}(\mathrm{MW} /$ day $)$ & 243 & 478 & 824 \\
\hline$\sum_{k \in \mathbb{D G}} R_{k}^{\mathrm{G}-}(\mathrm{MW} /$ day $)$ & 382 & 382 & 382 \\
\hline $\begin{array}{c}\text { Total expected deployed reserve } \\
(\mathrm{MWh} / \text { day })\end{array}$ & $2{ }^{\prime} 083$ & $2{ }^{\prime} 237$ & $2{ }^{\prime} 473$ \\
\hline
\end{tabular}

Table III. Reduction in the cost and ELNS of the grid due to ARADSs.

\begin{tabular}{c||c|c|c}
\hline VOLL (CHF/MWh) & 200 & 400 & 600 \\
\hline $\begin{array}{c}\text { Reduction in total expected cost } \\
\text { of TSO (CHF/day) }\end{array}$ & $72^{\prime} 080$ & $119^{\prime} 410$ & $176^{\prime} 650$ \\
\hline Reduction in ELNS (MWh/day) & 89 & 284 & 290 \\
\hline
\end{tabular}

injection at each bus of the transmission system from its dayahead scheduled value. This approach is utilized to model the contingencies and uncertainties by generating 20'000 scenarios. Finally, the k-medoids clustering method is applied to reduce the number of scenarios to 200 representative scenarios.

\section{Impact of Value of Lost Load (VOLL) on the Amount of} Allocated Reserve

The problem is modelled using YALMIP-MATLAB [20] and solved with GUROBI [21] on a PC with Windows equipped with a $2.8 \mathrm{GHz}$ Xeon CPU and $32 \mathrm{~GB}$ of RAM. Then, the optimal reserve allocation problem (1)-(21) is solved for 2 different cases. In case 1, both DBPPs and ARADSs provide active power reserve, while in case 2 , only DBPPs provide active power reserve. Table I and Table II indicate the impact of the chosen VOLL on the total cost and amount of the allocated reserves for cases 1 and 2, respectively. The total energy consumption of the Swissgrid network during the 24 hour of study is $144.2 \mathrm{GWh}$. The results show that, by increasing VOLL, the total booked/deployed reserve of TSO and its total cost increase while its ELNS decreases. The execution time of the proposed optimal allocation problem is less than 600 seconds for both cases. Due to the lack of space, detailed results are only shown for case 1 with VOLL equal to $400 \mathrm{CHF} / \mathrm{MWh}$ in Fig. 5 and Fig. 6, which respectively show the required power and energy reserve capacities from all providers.

\section{Technical and Economical benfits of the ARADS}

In order to achieve a clear perception about the economical and technical benefits of ARADSs, a comparison between cases 1 and 2 is reported in Table III. It denotes that the 
provision of active power reserve by ARADSs not only decreases the total expected cost of TSO, but it also improves the ELNS of TSO.

\section{CONCLUSIONS}

This paper proposes a method to allocate the required active power reserve of TSO for a desired scheduling time horizon while considering the capability of an emerging power system entity termed ARADS for provision of reserve. With reliance on the DC power flow model and a cost-benefit approach, it developed a linear scenario-based optimization formulation with the aim of minimizing the total cost of the TSO including the expected cost of load not served and the expected cost of reserve allocation.

The high-voltage transmission system of Switzerland, operated by Swissgrid, was used as a case study to show the performance and the effectiveness of the proposed method. A 24-hour scheduling time horizon with 24 1-hour dispatching time intervals was chosen to allocate the required active power reserve of Swissgrid network for the next day. The results showed that the method was able to compute the required active power reserve for the whole scheduling horizon in less than 600 seconds. The proposed method is designed to support TSOs to identify their needs for active power reserve from all DBPPs and ARADSs. In this respect, a method of this kind can facilitate a tighter and reliable cooperation between TSOs and lower-grid level operators and enable the transition from the current top-down mechanism for ancillary services procurement to a bidirectional approach. It was proved that this transition improves the electric power system security of supply against contingencies and reduces the cost of ancillary services by diversifying the ancillary services providers and increasing competition between them.

\section{ACKNOWLEDGMENT}

The authors thank Dr. Walter Sattinger, Dr. Jonas Muehlethaler and Dr. Marek Zima for granting us access to the technical data of Swissgrid's network and providing useful advices. This project is carried out within the frame of the Swiss Centre for Competence in Energy Research on the Future Swiss Electrical Infrastructure (SCCER-FURIES) with the financial support of the Swiss Innovation Agency (Innosuisse - SCCER program) and Swissgrid.

\section{REFERENCES}

[1] P. D. Redondo, and O. V. Vliet. "Modelling the energy future of Switzerland after the phase out of nuclear power plants." Energy Procedia, vol. 76, pp. 49-58, 2015.

[2] Y. G. Rebours, D. S. Kirschen, M. Trotignon, and S. Rossignol, "A survey of frequency and voltage control ancillary services-part I: Technical features," IEEE Trans on power syst., vol. 22, no. 1, pp. 350-357, 2007.

[3] Y. G. Rebours, D. S. Kirschen, M. Trotignon, and S. Rossignol, “A survey of frequency and voltage control ancillary services-part II: Economic features," IEEE Trans. on power syst., vol. 22, no. 1, pp. 358366, 2007.

[4] M. D. Galus, S. Koch, and G. Andersson, "Provision of load frequency control by PHEVs, controllable loads, and a cogeneration unit," IEEE Trans. on Industrial Electronics, vol. 58, no. 10, pp. 4568-4582,2011.

[5] V. C. Gungor, D. Sahin, T. Kocak, S. Ergut, C. Buccella, C. Cecati, and G. P. Hancke, "Smart grid technologies: Communication technologies and standards," IEEE trans. on Industrial informatics, vol. 7, no. 4, pp. 529539, 2011.

[6] N. Troy, D. Flynn, and M. O'Malley, "Multi-mode operation of combined cycle gas turbines with increasing wind penetration," IEEE Trans. on Power Syst., vol. 27, no. 1, pp. 484-492, 2012.

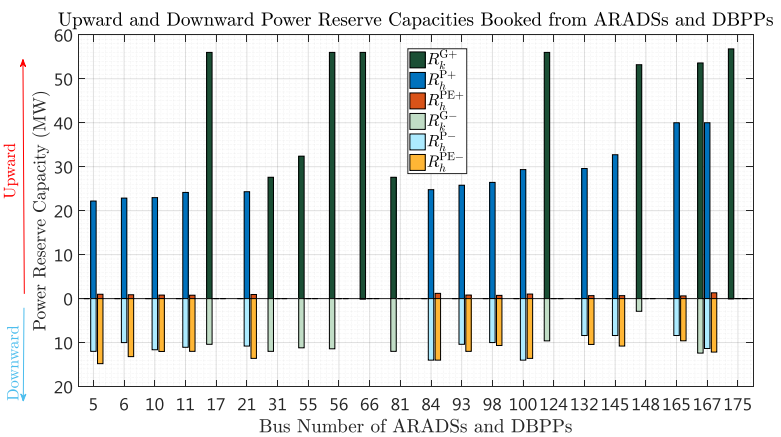

Fig. 5. Booked power reserve capacities from ARADSs and DBPPs for case 1 with $\mathrm{VOLL}=400 \mathrm{CHF} / \mathrm{MWh}$.

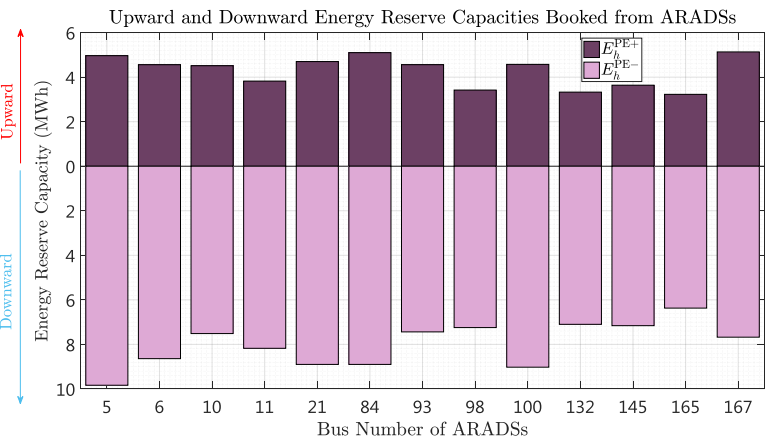

Fig. 6. Booked energy reserve capacities from ARADSs for case 1 with VOLL $=400 \mathrm{CHF} / \mathrm{MWh}$.

[7] A. J. Wood and B. F. Wollenberg, "Power Generation, Operation and Control", John Wiley \& Sons, 2012.

[8] R. Allan and R. Billinton, "Reliability evaluation of power systems". Springer Science \& Business Media, 2013.

[9] R. Doherty and M. O'malley, "A new approach to quantify reserve demand in systems with significant installed wind capacity," IEEE Trans. on Power Syst., vol. 20, no. 2, pp. 587-595, 2005.

[10] C. Maurer, S. Krahl, and H. Weber, "Dimensioning of secondary and tertiary control reserve by probabilistic methods," International Trans. on Elect. Energy Syst., vol. 19, no. 4, pp. 544-552, 2009.

[11] R. Ferrero and M. Shahidehpour, "Optimal reserve allocation and pricing," in Power Engineering Society General Meeting, 2003.

[12] A. Ahmadi-Khatir, M. Bozorg, R. Cherkaoui, "Probabilistic Spinning Reserve Provision Model in Multi-Control Zone Power System," IEEE Trans. on Power Syst., vol. 28, no. 3, pp. 2819-2829, Aug. 2013.

[13] J. M. Morales, A. J. Conejo, and J. Pérez-Ruiz, "Economic valuation of reserves in power systems with high penetration of wind power," IEEE Trans. on Power Syst., vol. 24, no. 2, pp. 900-910, 2009.

[14] M. A. Ortega-Vazquez, and D. S. Kirschen. "Optimizing the spinning reserve requirements using a cost/benefit analysis." IEEE Trans. on Power Syst., vol. 22, no. 1, pp. 24-33, Feb. 2007.

[15] M. Kalantar-Neyestanaki, M. Bozorg, F. Sossan, R. Cherkaoui, "Allocation of Frequency Control Reserve from Aggregated Resources of Active Distribution Systems”. In Power Syst. Computation Conf., Dublin, Ireland, June, 2018.

[16] Available: https://www.swissgrid.ch

[17] "Generating Unit Statistical Brochures 4 2013-2017 all units reporting," NERC, Aug. $2018 . \quad$ Available: https://www.nerc.com/pa/RAPA/gads/Reports.

[18] "Nordic and Baltic grid disturbance statistics 2015," ENTSOE, Brussels, Belgium, Jan. 2017 Available: https://docstore.entsoe.eu/Documents.

[19] M. Bozorg, F. Sossan, J. Y. Le Boudec, and M. Paolone, "Influencing the bulk power system reserve by dispatching power distribution networks using local energy storage". Elect. Power Syst. Res., 163, pp.270-279, June 2018.

[20] J. Löfberg, "YALMIP: A toolbox for modeling and optimization in MATLAB," in Proc. CACSD Conf., Taipei, Taiwan, pp. 284-289, 2004.

[21] "Gurobi Optimizer Reference Manual," Gurobi Optimization, Inc. [Online]. Available: http://www.gurobi.com. 